University of Nebraska - Lincoln

DigitalCommons@University of Nebraska - Lincoln

Faculty Publications, Department of Mathematics

Mathematics, Department of

$9-2006$

\title{
Robustness With Respect to Sampling for Stabilization of Riesz Spectral Systems
}

Richard Rebarber

University of Nebraska - Lincoln, rrebarber1@unl.edu

Stuart Townley

University of Exeter, Exeter EX4 4QE, U.K.

Follow this and additional works at: https://digitalcommons.unl.edu/mathfacpub

Part of the Mathematics Commons

Rebarber, Richard and Townley, Stuart, "Robustness With Respect to Sampling for Stabilization of Riesz Spectral Systems" (2006). Faculty Publications, Department of Mathematics. 31.

https://digitalcommons.unl.edu/mathfacpub/31

This Article is brought to you for free and open access by the Mathematics, Department of at DigitalCommons@University of Nebraska - Lincoln. It has been accepted for inclusion in Faculty Publications, Department of Mathematics by an authorized administrator of DigitalCommons@University of Nebraska - Lincoln. 


\section{Robustness With Respect to Sampling for Stabilization of Riesz Spectral Systems}

\author{
Richard Rebarber and Stuart Townley
}

\begin{abstract}
We suppose that a continuous-time feedback is input-output stabilizing for an infinite-dimensional system. We address the question of whether the sampled-data controller obtained by applying idealized sample-and-hold to this continuous-time feedback is also input-output stabilizing if the sampling time is small enough. This question has been previously addressed for fairly general systems under various conditions. In this note, we restrict our attention to Riesz spectral systems, for which we generalize the existing results. Specifically, we give two relatively simple conditions which, combined, are sufficient for the sampled-data controller to be stabilizing. The first condition is a spectrum decomposition for the open-loop system generator, which by itself is necessary, but not sufficient, for the system to be stabilizable by sampled-data control. The second is a summability condition relating the real part of the spectrum of the generator and the expansion coefficients for the input and feedback operators.
\end{abstract}

Index Terms-Infinite-dimensional systems, sampled-data control, stabilization.

\section{INTRODUCTION}

In this note, we consider systems of the form

$$
\begin{aligned}
& \dot{x}(t)=A x(t)+B u(t), \quad x(t) \in X, \quad u(t) \in \mathbb{C} \\
& y(t)=F x(t), \quad y(t) \in \mathbb{C}
\end{aligned}
$$

where $X$ is a complex and separable Hilbert space. We assume that $A$ is the (possibly unbounded) generator of a strongly continuous semigroup $T(t), t \geq 0$ on $X$, with eigenvalues $\lambda_{k}, k=1,2, \ldots$, and a Riesz basis of associated eigenvectors $\phi_{k}$. For Hilbert spaces $X$ and $Y$, let

Manuscript received October 13, 2004; revised February 22, 2006. Recommended by Associate Editor H. Hjalmarsson. This work was supported in part by the EPSRC under Grant GR/S01580/01 and by the National Science Foundation under Grant DMS-0206951.

R. Rebarber is with the Department of Mathematics, University of NebraskaLincoln, Lincoln, NE 68588-0130 USA (e-mail: rrebarbe@ @math.unl.edu).

S. Townley is with the School of Engineering, Computer Science, and Mathematics, Department of Mathematical Sciences, University of Exeter, Exeter EX4 4QE, U.K. (e-mail: townley @ maths.ex.ac.uk).

Digital Object Identifier 10.1109/TAC.2006.880797
$\mathcal{B}(X, Y)$ denote bounded operators from $X$ into $Y$. We assume that $B \in \mathcal{B}\left(\mathbb{C}, X_{-1}\right)$ and $F \in \mathcal{B}(X, \mathbb{C})$. Here, $X_{-1}$ is the completion of $X$ with respect to the norm $\|\cdot\|_{-1}$, where $\|x\|_{-1}:=\left\|(A-\rho)^{-1} x\right\|$, for any $\rho$ in the resolvent set of $A$. We define the scalars $f_{k}$ and $b_{k}$ via

$$
f_{k}=F \varphi_{k} \quad \text { and } \quad B=\sum_{k} b_{k} \varphi_{k}
$$

Without loss of generality, we can assume that $X=l^{2}(\mathbb{C})$ with index set $\mathbb{N}$. The condition that $B \in \mathcal{B}\left(\mathbb{C}, X_{-1}\right)$ is very general and here is is equivalent to

$$
\sum_{n=1}^{\infty}\left(\frac{\left|b_{k}\right|}{1+\left|\lambda_{k}\right|}\right)^{2}<\infty
$$

In Remark 2.5, we discuss the generalization of our results to systems where $\mathbb{C}$ is replaced by $\mathbb{C}^{m}$, but for the sake of clarity of exposition the proofs are given in the single-input-single-output case.

In [3] and [5], we considered a natural question concerning sampled-data stabilization of infinite-dimensional systems: If unity output feedback $u=y$ is stabilizing for the continuous-time system (1.1), is its digital implementation also stabilizing? More precisely, is the idealized sample and hold feedback

$$
u(t)=y(n \tau) \quad \text { for } \quad t \in[n \tau,(n+1) \tau)
$$

stabilizing for (1.1) if $\tau>0$, the sampling period, is small enough? This was shown in [2] to be true when $X$ is finite-dimensional, and would seem reasonable for infinite-dimensional systems. In [3], we showed that this is true for two large classes of infinite-dimensional systems. For one class, we allow arbitrary generators $A$, but require $B$ to be bounded and $F$ to be compact; in another class we allow $B$ to be highly unbounded but require $A$ to generate an analytic semigroup and $F$ to be compact. In the context of the single-input-single-output Riesz spectral systems as described before:

- Systems in the first class have $\left\{b_{k}\right\} \in l^{2}$ and $\left\{f_{k}\right\} \in l^{2}$, which in turn implies that

$$
\sum_{k=1}^{\infty}\left|b_{k} f_{k}\right|<\infty
$$

- Systems in the second class have

$$
\lambda_{k}=O\left(\left|\operatorname{Re} \lambda_{k}\right|\right) \quad(T(t) \text { is analytic }) .
$$

If either (1.4) or (1.5) hold, then

$$
\sum_{k} \frac{\left|b_{k} f_{k}\right|}{1+\left|\operatorname{Re} \lambda_{k}\right|}<\infty .
$$

When $X$ is infinite-dimensional, there are many systems of the form (1.1) which can be stabilized by $u=y$ but cannot be stabilized by sampled-data control. A result from [6] states that if a system can be stabilized by sampled-data control (even in open loop), then a number of restrictive necessary conditions must hold. In particular the operator $A$ must satisfy a spectral decomposition property and additionally have only a finite-dimensional unstable part. In the context of the systems 
under consideration in this note, assuming without loss of generality that $\left\{\operatorname{Re} \lambda_{k}\right\}$ is nondecreasing, this means that we can find $\beta>0$ and $\kappa \in \mathbb{N}$ so that

$$
\begin{aligned}
& \operatorname{Re} \lambda_{k}<-\beta \text { for all } k \geq \kappa \text { and } \\
& \operatorname{Re} \lambda_{k} \geq 0, \quad \text { for all } k<\kappa .
\end{aligned}
$$

We show that (1.6) and (1.7) are sufficient conditions for (1.3) to be stabilizing if the sampling period is small enough. In [1], we have an example where (1.6) fails, but nevertheless (1.3) is stabilizing if the sampling period is small enough, which shows that (1.6) is not a necessary condition. Note that in general (1.7) is not a sufficient condition for (1.3) to stabilize (1.1) for all sufficiently small $\tau>0$, as the counterexample in [5] demonstrates.

\section{Stability Result}

We will focus on a spectral/frequency domain approach, and therefore at first we ignore the issue of whether the closed-loop feedback operator $A+B F$ generates a $C_{0}$-semigroup, and initially state the problem in input output terms. With this input-output approach in mind we define the continuous time open-loop transfer function

$$
G(s):=F(s I-A)^{-1} B .
$$

In the Riesz spectral case we are considering

$$
G(s)=\sum_{k=1}^{\infty} \frac{b_{k} f_{k}}{s-\lambda_{k}}
$$

Let

$$
x_{n}:=x(n \tau) .
$$

Applying (1.3) to (1.1) results in the discrete-time system

$$
x_{n+1}=T(\tau) x_{n}+\left(\int_{0}^{\tau} T(s) B d s\right) F x_{n}
$$

which in the case that $A$ is invertible (that is, $\lambda_{k} \neq 0$ for all $k$ ), leads to a discrete-time open-loop transfer function

$$
H_{\tau}(z):=F(z I-T(\tau))^{-1}(T(\tau)-I) A^{-1} B
$$

see [3], [4], or [6] for details. In the Riesz spectral case

$$
H_{\tau}(z)=\sum_{k=1}^{\infty} \frac{b_{k} f_{k}}{\lambda_{k}}\left(\frac{e^{\lambda_{k} \tau}-1}{z-e^{\lambda_{k} \tau}}\right) .
$$

If some $\lambda_{k}=0$, we replace the corresponding term in the aforementioned sum with $b_{k} f_{k} \tau /(z-1)$, which is sufficiently easy to deal with that we assume without loss of generality that all $\lambda_{k} \neq 0$.

Our continuous time stability assumption is that the closed-loop continuous-time system is input-output stable in the sense that there exists $\epsilon^{*} \in(0,1)$ so that

$$
|1-G(s)| \geq \epsilon^{*} \quad \text { for all } \operatorname{Re}(s) \geq 0 .
$$

This implies that the continuous time closed-loop transfer function is input-output stable. In [3], [4], and [6], we showed that power stability of the discrete-time system (2.2) is equivalent to the exponential stability of the sampled data system (1.1) and (1.3). The discrete-time system is input-output stable if there exists $\epsilon \in(0,1)$ such that

$$
\left|1-H_{\tau}(z)\right|>\epsilon \text { for all }|z| \geq 1
$$

This is what we prove, although in Corollary 2.3 we translate this to state-space stability.

Theorem 2.1: Suppose that unity output feedback is input-output stabilizing for (1.1), by which we mean there exists $\epsilon^{*} \in(0,1)$ so that (2.4) holds. If (1.6) and (1.7) hold, then for all $\epsilon \in\left(0, \epsilon^{*}\right)$, there exists $\tau^{*}>0$ such that for every $\tau \in\left(0, \tau^{*}\right)(2.5)$ holds.

Proof: We establish (2.5) by using (2.4) and an approximation argument. We divide the proof up into a number of steps. In steps 1) and 2), we look at the infinite-dimensional tails in the transfer functions $G(s)$ and $H_{\tau}(z)$ and show that they are small in some appropriate sense. In step 3), we look at the finite-dimensional truncation

$$
1-\sum_{k=1}^{K_{2}-1} b_{k} f_{k} \frac{e^{\lambda_{k} \tau}-1}{\lambda_{k}\left(z-e^{\lambda_{k} \tau}\right)}
$$

for $|z| \geq 1$ but sufficiently close to 1 . In step 4) we look at the same truncated transfer function, but this time bounded away from 1. In both steps 3 ) and 4), we rely heavily on a comparison between truncations of the continuous- and discrete-time transfer functions $G(s)$ and $H_{\tau}\left(e^{s \tau}\right)$. Step 5) pulls steps 1)-4) together.

Step 1) We consider the infinite-dimensional tail in the continuous time transfer function $G(s)$. If $s \in \mathbb{C}_{0}:=\{s \in$ $\mathbb{C} \mid \operatorname{Re}(s)>0\}$ and $K \geq \kappa$, then using (1.7)

$$
\sum_{k=K}^{\infty}\left|\frac{b_{k} f_{k}}{s-\lambda_{k}}\right| \leq \sum_{k=K}^{\infty}\left|\frac{b_{k} f_{k}}{\operatorname{Re} \lambda_{k}}\right|
$$

Now using (1.6) it follows easily that there exists a large enough $K_{1} \geq \kappa$ so that for all $K \geq K_{1}$

$$
\sup _{s \in \mathbb{C}_{0}} \sum_{k=K}^{\infty}\left|\frac{b_{k} f_{k}}{s-\lambda_{k}}\right|<\frac{\epsilon^{*}-\epsilon}{2} .
$$

Step 2) Next we similarly consider the infinite-dimensional tail in the discrete-time transfer function $H_{\tau}(z)$. We claim that there exists $K_{2}>K_{1} \geq \kappa$ such that for all $z \in \mathbb{C}$ with $|z| \geq 1$

$$
\sum_{k=K_{2}}^{\infty}\left|\frac{b_{k} f_{k}}{\lambda_{k}}\left(\frac{e^{\lambda_{k} \tau}-1}{z-e^{\lambda_{k} \tau}}\right)\right|<\frac{\epsilon^{*}-\epsilon}{4} .
$$

To see this, we first rewrite the summand in (2.7) so that

$$
\sum_{k=K_{2}}^{\infty}\left|\frac{b_{k} f_{k}}{\lambda_{k}}\left(\frac{e^{\lambda_{k} \tau}-1}{z-e^{\lambda_{k} \tau}}\right)\right|=\sum_{k=K_{2}}^{\infty}\left|\frac{b_{k} f_{k}}{\operatorname{Re} \lambda_{k}}\left(\frac{\frac{e^{\lambda_{k} \tau}-1}{\lambda_{k} \tau}}{\frac{z-e^{\lambda_{k} \tau}}{\operatorname{Re} \lambda_{k} \tau}}\right)\right| .
$$

Looking at the summand in the right-hand side of (2.8), remembering that $\operatorname{Re} \lambda_{k}<0$ for $k \geq \kappa$, and using $|z| \geq 1$, we see that

$$
\left|\frac{\frac{e^{\lambda_{k} \tau}-1}{\lambda_{k} \tau}}{\frac{z-e^{\lambda_{k} \tau}}{\operatorname{Re} \lambda_{k} \tau}}\right| \leq \frac{\left|\frac{e^{\lambda_{k} \tau}-1}{\lambda_{k} \tau}\right|}{\frac{1-e^{\operatorname{Re} \lambda_{k} \tau}}{\left|\operatorname{Re} \lambda_{k} \tau\right|}} .
$$

Let $\delta>0$. We need to consider two cases. 
i) If $-\delta<\operatorname{Re}\left(\lambda_{k} \tau\right)<0$, then taking a Taylor series expansions yields

$$
\left|\frac{e^{\lambda_{k} \tau}-1}{\lambda_{k} \tau}\right|=O(1), \quad \frac{1-e^{\operatorname{Re} \lambda_{k} \tau}}{\left|\operatorname{Re} \lambda_{k} \tau\right|}=1+O(\delta) .
$$

ii) If $\operatorname{Re}\left(\lambda_{k} \tau\right)<-\delta$, then

$$
\left|\frac{\left(\frac{e^{\lambda_{k} \tau}-1}{\lambda_{k}}\right)}{\left(\frac{1-e^{\lambda_{k} \tau}}{\operatorname{Re} \lambda_{k}}\right)}\right|<\frac{2}{1-e^{-\delta}}\left|\frac{\operatorname{Re} \lambda_{k}}{\lambda_{k}}\right| \leq \frac{2}{1-e^{-\delta}} .
$$

Combining cases i) and ii) with (1.6) yields (2.7).

Step 3) In this step, we consider

$$
1-\sum_{k=1}^{K_{2}-1} b_{k} f_{k} \frac{e^{\lambda_{k} \tau}-1}{\lambda_{k}\left(z-e^{\lambda_{k} \tau}\right)}
$$

for those $z$ with $|z| \geq 1$ which are sufficiently close to $z=1$ by making a comparison to the corresponding continuous-time truncated transfer function

$$
1-\sum_{k=1}^{K_{2}-1} \frac{b_{k} f_{k}}{s-\lambda_{k}}
$$

To do this, we use a parametrization of $z$ in terms of $s$ and $\tau$ (as in [3]). Indeed, using the analyticity of $e^{z}$ and the open mapping theorem we can write such $z$ as $z=e^{s \tau}$, with $\operatorname{Re}(s)>0,|s \tau|<\eta$ and $\eta>0$ sufficiently small. We accordingly define

$$
S=\left\{z=e^{s \tau}|\operatorname{Re} s>0,| s \tau \mid<\eta\right\}
$$

and then compare the truncated discrete-time function evaluated at $z \in S$ to the continuous-time transfer function evaluated at the corresponding $s$.

We first write

$$
\begin{aligned}
\left|1-\sum_{k=1}^{K_{2}-1} b_{k} f_{k} \frac{e^{\lambda_{k} \tau}-1}{\lambda_{k}\left(z-e^{\lambda_{k} \tau}\right)}\right|=\mid 1-\sum_{k=1}^{K_{2}-1} \frac{b_{k} f_{k}}{s-\lambda_{k}} \\
+\sum_{k=1}^{K_{2}-1} b_{k} f_{k}\left(\frac{1}{s-\lambda_{k}}-\frac{e^{\lambda_{k} \tau}-1}{\lambda_{k}\left(z-e^{\lambda_{k} \tau}\right)}\right) \mid
\end{aligned}
$$

and rearrange the third term on the right-hand side to give

$$
\begin{aligned}
& \sum_{k=1}^{K_{2}-1} b_{k} f_{k}\left(\frac{1}{s-\lambda_{k}}-\right.\left.\frac{e^{\lambda_{k} \tau}-1}{\lambda_{k}\left(z-e^{\lambda_{k} \tau}\right)}\right) \\
&=\sum_{k=1}^{K_{2}-1} \frac{b_{k} f_{k}}{s-\lambda_{k}}\left(1-\frac{\frac{e^{\lambda_{k} \tau}-1}{\lambda_{k} \tau}}{\frac{e^{s \tau-e^{\lambda_{k} \tau}}}{\left(s-\lambda_{k}\right) \tau}}\right)
\end{aligned}
$$

Now, for sufficiently small $\tau$ and $\eta$ we have

$$
\begin{aligned}
1-\frac{\frac{e^{\lambda_{k} \tau}-1}{\lambda_{k} \tau}}{\frac{e^{s \tau-e^{\lambda_{k} \tau}}}{\left(s-\lambda_{k}\right) \tau}} & =1-\frac{\frac{e^{\lambda_{k} \tau}-1}{\lambda_{k} \tau}}{\frac{\left(e^{\left(\lambda_{k}-s\right) \tau}-1\right) e^{s \tau}}{\left(\lambda_{k}-s\right) \tau}} \\
& =1-\frac{1+O\left(\lambda_{k} \tau\right)}{\left[1+O\left(\left(s-\lambda_{k}\right) \tau\right)\right][1+O(s \tau)]}
\end{aligned}
$$

Here, we used the fact that $\lambda_{k} \tau$ is small for all small enough $\tau$, since we have only finitely many $\lambda_{k}$, and that from the definition of $S$ we have $|s \tau|<\eta$. It follows that on $S$ we have that

$$
\lim _{(\tau, \eta) \rightarrow 0}\left(1-\frac{\frac{e^{\lambda_{k} \tau}-1}{\lambda_{k} \tau}}{\frac{e^{s \tau}-e_{k} \tau}{\left(s-\lambda_{k}\right) \tau}}\right)=0
$$

It would now seem reasonable to conclude that the truncated discrete-time transfer function approximates the corresponding truncated continuous-time transfer function arbirarily closely for all $z \in S$ and all sufficiently small $\tau$ and $\eta$. There is one delicate issue: for $z \in S$ the corresponding $s$ could take values arbitrarily close to the unstable eigenvalues $\lambda_{k}$, where the transfer functions approach arbitrarily large values. Obviously, if no $\lambda_{k}$ is unstable, i.e. $\kappa=1$, then we are done. We assume therefore that $\kappa>1$ and divide the subsequent analysis into two cases.

- Step 3a) We first consider those $z \in S$, so that the corresponding $s$ is close to one of the unstable $\lambda_{k}$, i.e., for $k=1, \ldots, \kappa-1$. Let this be $\lambda_{k} *$ and suppose, specifically, that $\left|s-\lambda_{k}\right|<a$, where $a \in(0, \alpha)$ and $\alpha>0$ is the minimum separation of the unstable $\lambda_{k}$. Then

$$
\left|\frac{b_{k *} f_{k *}}{s-\lambda_{k *}}\right| \geq \frac{\left|b_{k^{*}} f_{k *}\right|}{a}
$$

If the mode $\lambda_{k} *$ is uncontrollable or unobservable, then this term is zero and so it can be ignored. If it is controllable and observable, then it is large. In this latter case, using the previous argument that

$$
\frac{1}{s-\lambda_{k}} \approx\left(\frac{e^{\lambda_{k} \tau}-1}{\lambda_{k}\left(z-e^{\lambda_{k} \tau}\right)}\right)
$$

it follows that by choosing $a, \tau$ and $\eta$ small enough, we can ensure that for some $\mu>\epsilon^{*}$

$$
\begin{aligned}
& \mid 1-b_{k *} f_{k *} \frac{e^{\lambda_{k} * \tau}-1}{\lambda_{k *}\left(z-e^{\lambda_{k} * \tau}\right)}- \\
& \sum_{k=1, k \neq k^{*}}^{K_{2}-1} b_{k} f_{k} \frac{e^{\lambda_{k} \tau}-1}{\lambda_{k}\left(z-e^{\lambda_{k} \tau}\right)} \mid \geq \mu>\epsilon^{*} .
\end{aligned}
$$

- Step 3b) If $z \in S$, but the corresponding $s$ satisfies $\mid s-$ $\lambda_{k} \mid \geq a$ for $k=1, \ldots \kappa-1$, then all of the summands in

$$
\sum_{k=1}^{K_{2}-1} \frac{b_{k} f_{k}}{s-\lambda_{k}}
$$

are bounded. Using (2.9)-(2.11), it then follows that for all small enough $\tau$ and $\eta$

$$
\left|1-\sum_{k=1}^{K_{2}-1} b_{k} f_{k} \frac{e^{\lambda_{k} \tau}-1}{\lambda_{k}\left(z-e^{\lambda_{k} \tau}\right)}\right| \geq\left|1-\sum_{k=1}^{K_{2}-1} \frac{b_{k} f_{k}}{s-\lambda_{k}}\right|-\frac{\epsilon^{*}-\epsilon}{4} .
$$


Therefore, combining (2.13) with (2.4), (2.6), and (2.7), we have using $K_{2} \geq K_{1} \geq \kappa$ that

$$
\begin{aligned}
& \left|1-H_{\tau}(z)\right| \stackrel{(2.7)}{\geq}\left|1-\sum_{k=1}^{K_{2}-1} b_{k} f_{k} \frac{e^{\lambda_{k} \tau}-1}{\lambda_{k}\left(z-e^{\lambda_{k} \tau}\right)}\right|-\frac{\epsilon^{*}-\epsilon}{4} \\
& \stackrel{(2.4,2.6,2.13)}{\geq} \epsilon^{*}-\frac{\epsilon^{*}-\epsilon}{2}-\frac{\epsilon^{*}-\epsilon}{4}-\frac{\epsilon^{*}-\epsilon}{4} \\
& =\epsilon \text {. }
\end{aligned}
$$

Step 4) All that remains is to consider those $z$, with $|z| \geq 1$, that are bounded away from 1 . More precisely, choose $\gamma$ sufficiently small so that if $|z| \geq 1$ and $|z-1|<\gamma$, then $z \in S$, where $S$ has been fixed by the choices of sufficiently small $\tau$ and $\eta$ in step 3).

Now, consider those $z$ with $|z| \geq 1$ and $|z-1| \geq \gamma$. We have that

$$
\begin{aligned}
1-H_{\tau}(z)=1-\sum_{k=0}^{K-1} \frac{b_{k} f_{k}}{\lambda_{k}}\left(\frac{e^{\lambda_{k} \tau}-1}{z-e^{\lambda_{k} \tau}}\right) & \\
& +\sum_{k=0}^{K-1} \frac{b_{k} f_{k}}{\lambda_{k}}\left(\frac{e^{\lambda_{k} \tau}-1}{z-e^{\lambda_{k} \tau}}\right)-H_{\tau}(z) .
\end{aligned}
$$

From Step 2), if $|z| \geq 1$, then

$$
\left|1-H_{\tau}(z)\right| \geq\left|1-\sum_{k=0}^{K-1} \frac{b_{k} f_{k}}{\lambda_{k}}\left(\frac{e^{\lambda_{k} \tau}-1}{z-e^{\lambda_{k} \tau}}\right)\right|-\frac{\epsilon^{*}-\epsilon}{4}
$$

Since $|z-1| \geq \gamma$, it is obvious that we can find $\tau^{*}$ sufficiently small so that if $\tau \in\left(0, \tau^{*}\right)$, then

$$
\left|1-\sum_{k=1}^{K_{3}-1} b_{k} f_{k} \frac{e^{\lambda_{k} \tau}-1}{\lambda_{k}\left(z-e^{\lambda_{k} \tau}\right)}\right| \geq \epsilon^{*}
$$

This is because the denominators of the terms in this finite sum are all bounded away from 0 whilst the corresponding numerators tend to 0 as $\tau$ tends to 0 .

Step 5) To summarize, piecing together Steps 1)-4), we have proved that for all $\epsilon \in\left(0, \epsilon^{*}\right)$, there exists $\tau^{*}>0$ such that for every $\tau \in\left(0, \tau^{*}\right),(2.5)$ holds, as claimed.

Remark 2.2: Closed-loop continuous time $H_{\infty}$-style performance is related to the $H_{\infty}$-norm of the sensitivity function $(I-G(s))^{-1}$, while closed-loop sampled-data performance is related to the $H_{\infty}$-norm of the sensitivity function $\left(I-H_{\tau}(s)\right)^{-1}$ of the related discrete time system. Theorem 2.1 then shows that the continuous time performance can be "recovered" in the sampled-data system by sampling fast enough.

We next show that if we include some mild conditions on $(A, B, F)$, then we can conclude that the sampled data system is exponentially stable.

Corollary 2.3: Suppose that $\kappa>1$, i.e., the open-loop system has an unstable part, and

1) for all $k=1, \ldots \kappa-1, b_{k} \neq 0$ and $f_{k} \neq 0$;

2) if $k \neq j$, then $\lambda_{k} \neq \lambda_{j}$;

3) (1.6) and (1.7) hold; and

4) there exists $\epsilon^{*}>0$ such that (2.4) holds.

Then, there exists $\tau^{*}>0$ such that for every $\tau \in\left(0, \tau^{*}\right)$, the closedloop sampled data system (1.1), (1.3) is exponentially stable, in the sense that there exists $N \geq 1$ and $\nu>0$ such that the solution of (1.1), (1.3) with $x(0)=x_{0}$ satisfies

$$
\|x(t)\| \leq N e^{-\nu t}\left\|x_{0}\right\|
$$

Proof: First note that hypotheses 1) and 2) guarantee that the unstable part of $(A, B, F)$ is controllable and observable. By Theorem 2.1, hypotheses 3) and 4) guarantee (2.5) for all small enough $\tau>0$. Hence, from the proof of lemma 4.7 in [3], these hypotheses are sufficient to conclude that

$$
\Delta_{\tau}:=T(\tau)+\int_{0}^{\tau} T(s) B F d s
$$

is power stable for all small enough $\tau>0$. Then, [3, Lemma 2.3] we see that (1.1) and (1.3) are exponentially stable.

Remark 2.4: It is very easy to construct examples which satisfy (1.6) but do not satisfy either of the sets of hypotheses in [3]. For instance, if $T(t)$ is a differentiable semigroup which is not analytic, then $\left\{\left|\operatorname{Re}\left(\lambda_{k}\right)\right|\right\}$ is an unbounded sequence, so there exist $b \notin \ell^{2}$ for which (1.6) is satisfied for any $f \in \ell^{2}(\mathbb{C})$. In fact, even if there exists $\alpha, \beta>0$ such that

$$
\alpha \leq\left|\operatorname{Re}\left(\lambda_{k}\right)\right| \leq \beta
$$

(so $T(t)$ is not analytic), for a given $f \in \ell^{2}$ there might exist $b \notin \ell^{2}$ (so $B$ is not bounded) for which (1.6) is satisfied. As a trivial example, if $f_{k}=1 / k$, we can take $b_{k}=1 / k^{1 / 4}$, so $b \notin \ell^{2}$ but (1.6) is satisfied.

Remark 2.5: Suppose that the control space is $\mathbb{C}^{m}, m \in \mathbb{N}, m>1$, rather than $\mathbb{C}$. Then, it is not hard to modify our results. In this case $b_{k} \in \mathcal{B}\left(\mathbb{C}^{m}, \mathbb{C}\right)$, i.e., $b_{k}$ is a row vector of length $m$. Similarly, $f_{k}$ is now a column vector of length $m$. The transfer function becomes

$$
G(s)=\sum_{k=1}^{\infty} \frac{f_{k} b_{k}}{s-\lambda_{k}}
$$

where the matrix $f_{k} b_{k}$ has norm $\left\|f_{k}\right\|_{\mathbb{C}^{m}}\left\|b_{k}\right\|_{\mathbb{C}^{m}}$. Theorem 2.1 is now true with (1.6) replaced by

$$
\sum_{k} \frac{\left\|b_{k}\right\|_{\mathbb{C}^{m}}\left\|f_{k}\right\|_{\mathbb{C}^{m}}}{1+\left|\operatorname{Re} \lambda_{k}\right|}<\infty
$$

The proof is the same, with $b_{k} f_{k}$ replaced by $\left\|f_{k}\right\|_{\mathbb{C}^{m}}\left\|b_{k}\right\|_{\mathbb{C}^{m}}$. Similarly, Corollary 2.3 is true, with the conditions that $b_{k} \neq 0$ and $f_{k} \neq 0$ for $k=1, \ldots \kappa-1$ replaced by conditions which guarantee that the unstable part of $(A, B, F)$ is controllable and observable.

\section{REFERENCES}

[1] J. Azzam, R. Rebarber, and J. Windle, "Sampled data feedback stabilization of a beam equation with boundary control," in Proc. 2004 MTNS Symp., Leuven, Belgium, July 5-9, 2004.

[2] T. Chen and B. Francis, "Input-output stability of sampled-data systems," IEEE Trans. Autom. Control, vol. 36, no. 1, pp. 50-58, Jan. 1991.

[3] H. Logemann, R. Rebarber, and S. Townley, "Stability of infinite-dimensional sampled-data systems," Trans. Amer. Math. Soc., vol. 355, pp. 3301-3328, 2003.

[4] R. Rebarber and S. Townley, "Stabilization of distributed parameter systems using piecewise polynomial control," IEEE Trans. Autom. Control, vol. 42, no. 9, pp. 1254-1257, Sep. 1997.

[5] — "Nonrobustness of closed-loop stability for infinite-dimensional systems under sample and hold," IEEE Trans. Autom. Control, vol. 47, no. 8, pp. 1381-1385, Aug. 2002.

[6] S. Townley, R. Rebarber, H. J. Zwart, and D. K. Oates, "Stabilization of infinite-dimensional systems by generalized sampled-data control," in Proc. 3rd Int. Symp. Methods and Models in Automation and Robotics, Miedzyzdroje, Poland, Sep. 10-13, 1996, pp. 127-132. 